\title{
Percepciones de Ecuador como Destino según el Turista que Viaja desde Guayaquil
}

\section{Perceptions of Ecuador as a Tourism Destination for those who Travel from Guayaquil}

Tito Alfredo Macías Clavijo ${ }^{a}$, Wilmer Ernesto Rivadeneira Laz ${ }^{b}$, Julio Enrique Gavilanes Valle ${ }^{c}$, Gabriela Maldonado Pérez ${ }^{d}$, Mateo Julián Estrella Durán ${ }^{e}$

INFORMACIÓN DEL ARTÍCULO

Fecha de recepción: 31 de marzo del 2017

Fecha de aceptación: 15 de junio de 2017

a Licenciado en Turismo. Escuela Superior Politécnica del Litoral, ESPOL, Facultad de Ingeniería Marítima, Ciencias Oceánicas, Biológicas y Recursos Naturales. E-mail: tamacias@espol.edu.ec

$b$ Licenciado en Turismo. Escuela Superior Politécnica del Litoral, ESPOL, Facultad de Ingeniería Marítima, Ciencias Oceánicas, Biológicas y Recursos Naturales. E-mail: wrivaden@espol.edu.ec

\begin{abstract}
Resumen
La infraestructura, modernización y crecimiento modular que ha experimentado el anterior aeropuerto Simón Bolívar, conocido ahora como José Joaquín de Olmedo, desde la inauguración de su nueva terminal en julio de 2006, ha permitido que muchas aerolíneas encuentren atractivo el hacer de éste, su centro de conexiones internacionales, lo que a su vez ha permitido que el incremento del número de llegadas y salidas de visitantes que arriban al Ecuador, se produzcan desde la ciudad de Guayaquil. A partir de una investigación de tipo descriptiva y cumpliendo las normas de la autoridad aeroportuaria, se aplicaron encuestas aleatorias a turistas extranjeros que salieron desde Guayaquil, luego de visitar Ecuador, encontrándose que muchos de ellos realizaron su viaje por actividades relacionadas con su tiempo libre o negocios. El resultado de este estudio permitió determinar su percepción, intereses, probabilidad de retorno, recomendación del destino y otros datos relacionados, sobre la experiencia previa y posterior de su visita al país.
\end{abstract}

Palabras Clave:

Imagen del destino, percepción, atractivo turístico, emociones, frecuencia.

Clasificación JEL: L83

69 


\section{Percepciones de Ecuador como Destino según el Turista que Viaja desde Guayaquil}

\section{Perceptions of Ecuador as a Tourism Destination for those who Travel from Guayaquil}

Tito Alfredo Macias Clavijo ${ }^{a}$, Wilmer Ernesto Rivadeneira Laz ${ }^{b}$, Julio Enrique Gavilanes Valle ${ }^{c}$, Gabriela Maldonado Pérez ${ }^{d}$, Mateo Julián Estrella Durán ${ }^{e}$

\footnotetext{
c Máster en Turismo y Gestión Hotelera. Escuela Superior Politécnica del Litoral, ESPOL, Facultad de Ingeniería Marítima, Ciencias Oceánicas, Biológicas y Recursos Naturales.

E-mail: julengav@espol.edu.ec
}

${ }^{d}$ Maestra en Políticas Públicas con Mención en Gestión del Desarrollo. Pontificia Universidad Católica del Ecuador, PUCE, Facultad de Ciencias Humanas, Escuela de Hotelería y Turismo.

E-mail: gmaldonado@puce.edu.ec

${ }^{e}$ Máster OMT GTAT en Alta Gestión En Política y Estrategia de los Destinos Turísticos. Universidad de Cuenca, Facultad de Ciencias de la Hospitalidad.

E-mail:mateo.estrella@ucuenca.edu.ec

\begin{abstract}
The infrastructure modernization and the modular growth experienced by the former Simón Bolívar airport, now known as José Joaquin de Olmedo, since the introduction of its new terminal in July 2006, have made it attractive for many airlines to establish the hub of their international operations at that airport. This, in turn, has increased the number of international visitors, who arrive and depart from Ecuador by way of the Guayaquil airport. Random surveys of airline passengers shows that, upon arrival, many of them leave Guayaquil and take tours to visit the rest of Ecuador and practice activities related to their free time or business. This study shows the interests, probability of return, perception, recommendations and other related data concerning their visit's experience.
\end{abstract}

Keywords

Destination image, perception, tourism attraction, emotions, frequency.

JEL Classification: L83 


\section{Introducción}

Ecuador es el país latinoamericano con la mayor tasa de crecimiento en el ingreso de turistas extranjeros desde el 2010 , con un balance positivo del $48.7 \%$ en esta materia, dato sustentando por la South American Hotel \& Tourism Investment Conference (SAHIC por sus siglas en inglés) durante la octava edición del evento (SAHIC, 2015).

Según el Ministerio de Turismo (2016), el gasto global que desembolsan los turistas foráneos que visitan Ecuador se estima en USD 1.200 millones aproximadamente y el objetivo de esa cartera de Estado es hacer del turismo la principal actividad económica no petrolera en 2018; para lograrlo se espera ofrecer servicios especializados, estimular las inversiones turísticas y fortalecer la promoción interna y externa del potencial turístico de la nación. Una de las últimas campañas publicitarias realizadas es la denominada "All you need is Ecuador", misma que fue realizada desde el 2015 y se puso en marcha en algunas ciudades de países emisores de turismo, así como dentro de Ecuador, con el objetivo de posicionar al país como destino turístico de clase mundial. Así mismo existió la campaña "Feel again", que contó con el auspicio de artistas internacionales que recorrieron Ecuador mostrando la diversidad de paisajes de las diferentes regiones del país, con esto se logró visibilizar al Ecuador en ciudades como París, Londres, Madrid y otras en las que se presentó la mencionada campaña.

Como uno de los ejes de desarrollo en el Ecuador, la ciudad de Guayaquil ha experimentado uno de los más grandes procesos de desarrollo urbano en Latinoamérica durante los últimos 15 años (Delgado, 2013). Desde 1992, la Municipalidad de Guayaquil ha operado bajo un concepto de gestión que favorezca la eficiencia basada en la planificación a largo plazo (Delgado \& Chávez, 2016) y en 2004, el Programa de las Naciones Unidas para el Desarrollo, PNUD, nombró a Guayaquil como la mejor ciudad administrada en América Latina (PNUD, 2013).

Guayaquil como puerta de entrada y salida para viajeros nacionales y extranjeros tiene en su haber al aeropuerto José Joaquín de Olmedo, que cuenta con más de 50 mil metros cuadrados de construcción y recibe 7 millones de pasajeros por año (TAGSA, 2016); su diseño y eficiencia en el servicio ha ocasionado que se lo considere entre las mejores terminales aéreas del mundo en su categoría (ACI, 2016). La Dirección General de Aviación Civil del Ecuador (DGAC) se encarga de sus operaciones aeronáuticas y aeroportuarias en la ciudad y el país (DGAC, 2017). Por su desempeño fue galardonado en el año 2011 como el mejor aeropuerto del mundo por su tamaño según el Consejo Internacional de Aeropuertos (Airports Council International, 2011), y en 2013 fue nombrado mejor aeropuerto de América por esta misma organización. Siendo un centro de arribo nacional e internacional la administración aeroportuaria ha implementado sistemas de gestión de calidad que se enfocan a priorizar al cliente y optimizar la eficacia de los servicios que ofrece. 
La competencia intraportuaria insta a las diferentes concesionarias del servicio a cambiar el modelo de planificación y administración para ser elegidos por los operadores aéreos ya sea como centro de interconexión o como salida o entrada del país (Jiménez et al., 2014), teniendo el Aeropuerto de Guayaquil su contraparte en la terminal aérea de Quito.

De acuerdo a las indicaciones del principal fabricante de aeronaves en el mundo, en su Instructivo de Planificador de Vuelo para aviones Boeing 787 (Boeing, 2014), el despegue en aeropuertos cercanos al nivel del mar, requiere de menor consumo de combustible para generar el empuje necesario para el despegue. La operación de motores a gran altura depende del flujo de aire, que se convierte en escaso para la generación de la potencia requerida, esto se transforma en una inyección de hasta $20 \%$ más que en altitudes inferiores a los 2000 pies (Miralles, 2015) sumado a que los vientos que fluctúan en la ciudad son calmados la mayor parte del año, esto es debido a la posición privilegiada de la ciudad en el Golfo de Guayaquil (Peralta, 2013). Esto transforma en un atractivo logístico la operación en la pista del aeropuerto José Joaquín de Olmedo, sumado a que su capacidad de crecimiento para recibir vuelos continuos y plataformas de embarque, están planificadas para aquello.

La terminal aeroportuaria es un espacio que permite el contacto comercial entre personas con diversos perfiles, incluyendo a turistas y visitantes, agentes comerciales y proveedores turísticos, , por tal motivo dentro de su área de cons- trucción, la terminal posee un centro de negocios y capacitación creado por Diamond Club cuyo propósito es el de ofrecer un lugar como instrumento de trabajo adicional.

Una innovación que presenta TAGSA según su página web es que en el aeropuerto labora personal bilingüe que garantiza una atención individualizada al visitante en inglés y español, así mismo indica que existen dos salas de conferencias con capacidad para 80 y 20 personas, respectivamente. Por otra parte, destaca que el aeropuerto de la ciudad tiene a disposición una oficina gerencial para ejecutivos que acuden a Guayaquil con necesidad de realizar negocios y que precisan de tener reuniones comerciales sin salir del aeropuerto, para lograr una optimización de su tiempo. Es debido a estas características del Terminal de Guayaquil, que un grupo importante de turistas la utilizan, como puerta de entrada y salida del Ecuador.

\section{Revisión Literaria}

Según el Ministerio de Turismo (2016) el 98.7\% de los turistas que llegan al país lo hacen por vía aérea; por tal motivo, un aeropuerto moderno se convierte en una herramienta decisiva que moviliza y dinamiza la economía de una nación. La mayoría de los turistas eligen un destino, incluido el aeropuerto, mediante el uso de plataformas que permiten comparar productos. Esto debido a que las tecnologías que usan los teléfonos móviles y las páginas web, permiten a los consumidores obtener información relacionada con sus viajes, 
así como la compra de productos turísticos básicos directamente de los proveedores de turismo (Morosan, 2014; Law, Leung, Lo, Leung, \& Hoc Nan Fong, 2015).

Las terminales aeroportuarias influyen en las diferentes actividades económicas de las ciudades donde se encuentran y además generan una primera $\mathrm{y}$ última visión de lo que representa un país, de ahí la necesidad de investigar la percepción de un destino que los viajeros poseen cuando se encuentran en este último punto de partida (Chen, 2012). Una terminal de aeropuerto, en particular un aeropuerto importante de un país, siempre ha sido tratado como el proyecto de la nación y la mayor parte del tiempo es una declaración arquitectónica icónica, ya que es la principal puerta de entrada al país (Mohd et al., 2016). Los turistas generan empatía con los destinos de acuerdo a su propia personalidad, y las características distintivas del destino se marcan en el contexto del tipo de turismo que se realiza, pues utilizan rasgos de la personalidad humana para reflejar al lugar visitado (Chen, 2012).

Los aeropuertos actualmente manejan tres formas de respuesta como mejora en sus ingresos: la primera, son las estrategias de marketing para sus consumidores primarios y secundarios; la segunda, es la forma en que el diseño de la terminal se ajusta a prestar las mayores facilidades para su uso; y la última, es la concepción de una metrópoli aeroportuaria que pueda ofrecer servicios de igual forma, solo que a menor escala que una ciudad (Díaz, 2015).
Además, un aeropuerto a menudo refleja las necesidades y requerimientos de sus grupos de interés, a través de sus departamentos y divisiones internas (Mohd et al., 2016).

Para captar la atención de extranjeros hacia Guayaquil, es importante forjar una imagen y reputación que cumpla con las expectativas de los visitantes. Una de las formas de fortalecer la imagen de Guayaquil como destino, es la implementación de un Plan de marketing, en especial con estrategias que capten la atención de turistas y toda la industria económicamente activa (Cruz, 2017). La imagen de un destino, se mide de acuerdo a las características que este posee, así como también a la capacidad de experimentación de emociones que los turistas son capaces de vivir, durante su estancia en el lugar visitado (Chen, 2012; Xiang, Magnini, \& Fesenmaier, 2014; Thakran \& Verma, 2013; Castells, 2011). Así mismo las emociones que los turistas son capaces de vivir van desde la impresión, encantamiento, positivismo, alegría, indiferencia, aburrimiento, desilusión, coraje, entre otras. De acuerdo a Chen (2012) las personas priorizan sus viajes en función de lo que esperan experimentar, es decir, si se desea vivir momentos de algarabía, el turista buscará destinos de fiestas y actividades, mientras que, si desea experimentar calma y paz, buscará destinos tranquilos, pues la experimentación tiene que ser compatible con la personalidad que refleja el destino y en adición, estos son esencialmente estereotipos del destino, cruciales en el proceso de toma de decisiones de los turistas (Di Marino, 2008). 
El perfil de los turistas, como la experiencia previa en un destino o la fuente de información, puede influir en la formación de su imagen de destino (Di Marino, 2008). Así mismo, analizar los antecedentes de la percepción turística, la imagen de destino y la satisfacción del turista puede proporcionar una visión en el proceso de creación de la lealtad de destino (Rajesh, 2013).

Debido a las restricciones en el tiempo, las finanzas personales, la compañía, o simplemente impulsado por las actitudes de novedad, los turistas no pueden elegir los destinos con la mayor predilección en mente. Como resultado, la intención de visitar puede parecer baja. Sin embargo, la relación post visita de los turistas con un destino puede reflejarse continuamente en su evaluación general del lugar, más allá de las fechas cercanas a la visita, es decir perdura en el tiempo (Zhang et al., 2014).

Anterior al hallazgo de Zhang (2014), se tiene que Chen (2012) menciona que la confianza influye directamente en el apego y afecta directa e indirectamente a la lealtad de los turistas por un destino. En comparación con la satisfacción y el apego, la confianza tiene un efecto más directo sobre la lealtad. Además, la confianza no sólo media la relación entre satisfacción y apego, sino también entre satisfacción y lealtad. Estos hallazgos sugieren que la confianza es la dimensión más importante de las variables y juega un papel muy importante en la construcción de un vínculo emocional entre turistas y destinos, como influir en las intenciones de comportamiento de los turistas
(Chen, 2012).

Para generar confianza y lealtad de turistas y mejorar su percepción hacia Ecuador, es necesario tratar de poner en marcha tipologías de turismo e implementar productos turísticos más atractivos y que busquen la participación activa de los diferentes grupos de interés, a su vez el turismo en el Ecuador es un sector con proyección en el corto y largo plazo y al tratarse de un destino no maduro, el país puede "aprender" de las experiencias llevadas a cabo en otros lugares (Castillo, 2015).

Adicional a las emociones que se deben experimentar y a la lealtad y confianza que se necesita generar, se debe contar con canales de compra eficientes ya que los actuales mecanismos de compra influyen en la experiencia al momento de elegir el destino, es decir, el canal genera un vínculo emocional con el consumidor, un ejemplo de esto son los blogs de viajeros que proporcionan una experiencia desde el usuario (Thakran \& Verma, 2013). Los hábitos del consumidor han cambiado en los últimos 25 años, al punto que el canal de venta y la selección emocional del destino van unidos, debido a la cantidad y calidad de información proporcionada; a esto se lo llama un modelo híbrido (Thakran \& Verma, 2013).

\section{Metodología}

La metodología empleada en esta investigación dentro del paradigma investigativo aplicado a las ciencias sociales es el positivismo, que en su base 
epistemológica según Barrantes (2000) es una percepción de la realidad objetiva que mide hechos sociales, opiniones o actitudes individuales. A su vez el método hipotético deductivo es conocido en un dominio de consideraciones teóricas que deben someterse a un escrutinio empírico (Bryman, 2012).

Es así que basados en las opiniones de los autores arriba mencionados se realizó un escrutinio de varias metodologías aplicadas que busquen medir la percepción del visitante hacia algún destino, lo que en definitiva lleva a encontrar la percepción de algún hecho social relevante (Barrantes, 2012). Al revisar detalladamente la literatura, se encontró que San Martín (2006) trabajó extensamente en temas de imágenes de destino, desarrollando un método de recolección de información con un cuestionario bastante completo para recopilar datos sobre la imagen de un destino, de tal forma que el cuestionario empleado en esta investigación está basado en el trabajo del mencionado autor.

Para esta investigación se usó como población de estudio a los usuarios de las instalaciones del aeropuerto José Joaquín de Olmedo de la ciudad de Guayaquil. La recolección de datos fue realizada durante el mes de septiembre de 2016, mes que coincide con el fin de la temporada alta de visitantes provenientes de países emisores del hemisferio norte y que según el Ministerio de Turismo, son el principal mercado al que el país apunta (MINTUR, 2014).

Para el cálculo de la muestra, se con- sideraron los datos proporcionados por el Ministerio de Turismo (2015) que indican la cantidad de visitantes extranjeros por nacionalidad recibidos en el Ecuador durante el año 2015, siendo este igual a 1'560.429 turistas a nivel nacional. Junto a este dato, se tiene que el $22 \%$ de turistas ingresan al país desde el aeropuerto internacional José Joaquín de Olmedo (MINTUR, 2015). Es así que, una vez realizados los cálculos, se tiene que aproximadamente 343.294 turistas arribaron a Ecuador por el aeropuerto de Guayaquil. Con esta cifra al aplicar un nivel de confianza del $93 \%$, un margen de error de $7 \%$ y un grado de respuesta de $50 \%$, se obtuvo que fuera necesario encuestar a 168 personas para ésta investigación. Cabe recalcar que se utilizó la fórmula de muestreo aleatorio simple, la cual se considera la forma más utilizada para realizar muestreos probabilísticos, pues con el muestreo aleatorio, cada unidad de la población tiene una probabilidad igual de inclusión en la muestra (Bryman, 2012).

Dado que esta investigación busca levantar la percepción del turista que usa el aeropuerto José Joaquín de Olmedo como puerta de salida del país, se aplicó un cuestionario estructurado a los encuestados, por tanto, la única pregunta que validaba la participación en el estudio era el lugar de residencia de las personas contactadas, de esta forma ecuatorianos y extranjeros residentes en el país no fueron considerados para continuar con la encuesta. El estudio se aplicó durante varios días en la sala de pre embarque internacional del aeropuerto. Debido a 
que la mayor cantidad de salidas internacionales se concentra en horas de la tarde y noche, muchas de las encuestas fueron recogidas a usuarios de vuelos que parten a estas horas. La obtención de los permisos para los encuestadores fue compleja y conllevó a que el personal participante, se someta a todas las políticas de seguridad exigidas para poder ingresar a una zona considerada como sensible en cualquier aeropuerto del mundo. De alguna forma esto se convirtió en una limitación de la investigación, pues se debió cumplir rigurosamente con los tiempos y horarios permitidos por la autoridad aeroportuaria para la toma de datos, quedando esta decisión fuera de la discrecionalidad de los investigadores. Esta rigurosidad obedece a que el aeropuerto de la ciudad, ha sido reconocido como uno de los mejores en la región, recibiendo distinciones a nivel latinoamericano e incluso mundial por su calidad y seguridad, para lo cual desde su concesión ha aplicado cánones de operación muy estrictos.

Como mencionó San Martín (2006), el instrumento usó variables cualitativas y para su medición se usaron escalas ordinales y nominales, con una serie de interrogantes que versaban sobre el motivo del viaje, tiempo de estadía, cantidad de dinero empleada en el viaje, consideración previa y posterior del destino elegido, valoración de la infraestructura turística y su grado de satisfacción sobre el destino.

\section{Resultados}

Los resultados se muestran a través de tablas de frecuencia, que tienen por ob- jeto proporcionar información referente al número de personas, porcentajes, y otras variables estudiadas a lo largo de este estudio.

La Tabla 1 resume algunas variables demográficas de los encuestados. En cuanto al género se observa una distribución más o menos proporcional de los encuestados, es así que los miembros del género masculino (54\%) es ligeramente mayor que el femenino (46\%). Referente a la edad de los encuestados, el rango de edades está dividido casi por igual, sin embargo, el rango de 25 a 34 años (33\%) muestra una diferencia superior con los otros grupos. Con respecto a la ocupación, los empleados privados representan el mayor grupo (54\%) y las amas de casa el menor (1\%).

Tabla 1.

Resumen del Perfil Demográfico

\begin{tabular}{ll}
\hline Género & Porcentaje \\
\hline Masculino & $54 \%$ \\
\hline Femenino & $46 \%$ \\
\hline Rango & Porcentaje \\
\hline 18 a 24 & $9 \%$ \\
\hline 25 a 34 & $33 \%$ \\
\hline 35 a 44 & $18 \%$ \\
\hline 45 a 54 & $11 \%$ \\
\hline 55 a 64 & $12 \%$ \\
\hline 65 o más & $17 \%$ \\
\hline Ocupación & Porcentaje \\
\hline Empleado público & $6 \%$ \\
\hline Empleado privado & $54 \%$ \\
\hline Desempleado & $1 \%$ \\
\hline Ama de casa & $1 \%$ \\
\hline Estudiante & $7 \%$ \\
\hline Jubilado & $21 \%$ \\
\hline Trabajador independiente & $5 \%$ \\
\hline Otros & $5 \%$ \\
\hline
\end{tabular}

76

PODIUM No. 31, Guayaquil, Junio 2017, pp. 69-86

Universidad Espíritu Santo - UEES

ISSN: 1390 - 5473 
En la figura 1 se observa el país de procedencia de los encuestados. Es así que se muestra que durante los días de toma de datos, la gran mayoría de los encuestados procedían de países euro- peos, siendo los holandeses quienes ocuparon el primer lugar, con una amplia diferencia sobre visitantes de otras naciones.

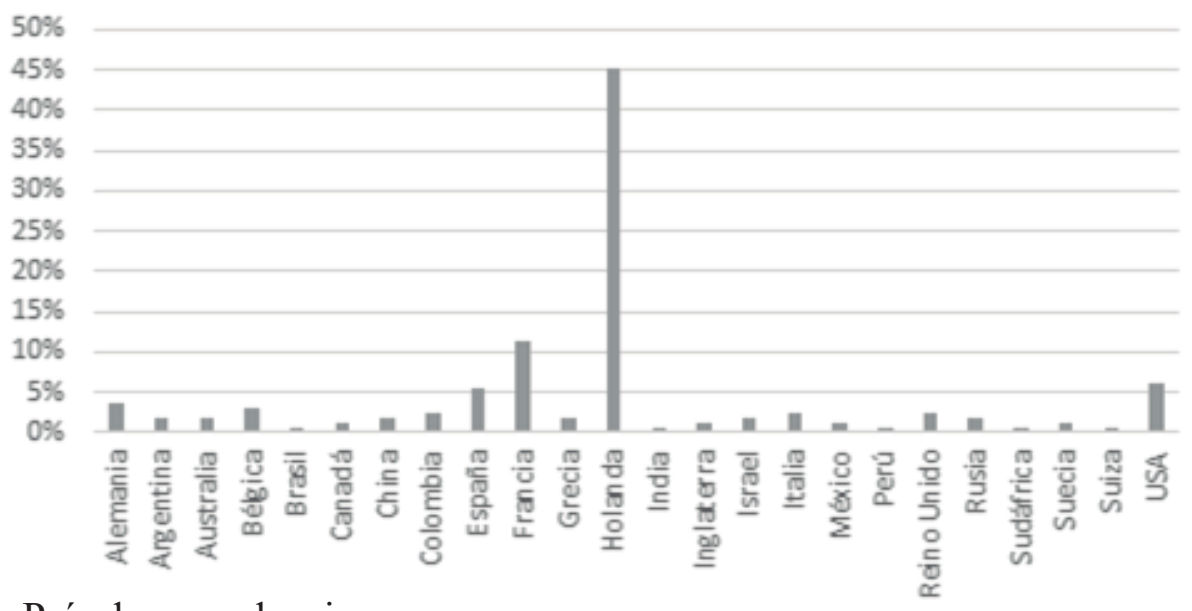

Figura 1. País de procedencia.

En cuanto a las visitas anteriores realizadas por los turistas hacia el Ecuador, sólo el 14\% reconoció haber visitado el país con anterioridad frente a un $86 \%$ que lo hacía por primera vez y la gran mayoría de los turistas que ya habían visitado el país, lo hizo por motivos de ocio (75\%), mientras que el resto lo hizo por negocios $(25 \%)$.

La tabla 2 muestra el gasto promedio y cantidad de días visitados al país, es así que un gran número de encuestados (47\%) señala que gastó en Ecuador entre 1000 y 3000 dólares de los Estados Unidos y así mismo permaneció aproximadamente 2 semanas en Ecuador.
Tabla 2.

Gasto promedio de los turistas y tiempo de permanencia

\begin{tabular}{ll}
\hline Gasto en destino & Porcentaje \\
Menos de 1000 USD & $40 \%$ \\
1000 a 3000 USD & $47 \%$ \\
3000 a 5000 USD & $10 \%$ \\
Más de 5000 USD & $3 \%$ \\
Tiempo en Ecuador & Porcentaje \\
Aprox. 1 semana & $16 \%$ \\
Aprox. 2 semanas & $52 \%$ \\
Aprox. 3 semanas & $23 \%$ \\
1 mes & $8 \%$ \\
Más de un mes & $1 \%$ \\
\hline
\end{tabular}


La tabla 3 señala las imágenes que acuden a la mente de los turistas encuestados cuando piensan en Ecuador como destino turístico. Los tres primeros lugares de imágenes asociadas al destino están ligadas a características naturales del país, siendo estas: paisajes únicos, flora y fauna; sol y playa, mientras que los últimos lugares lo ocuparon características negativas del destino como: insalubridad/enfermedades, baja conectividad del área y baja calidad de los servicios.

Tabla 3.

¿Con qué asocia el turista a Ecuador?

\begin{tabular}{ll}
\hline Ubicación & Características \\
\hline 1er lugar & Paisajes únicos \\
\hline 2do lugar & Flora y fauna \\
\hline 3er lugar & Sol y playa \\
\hline 4to lugar & Sitios Patrimoniales \\
\hline 5to lugar & Aventura \\
\hline 6to lugar & Gastronomía \\
\hline 7mo lugar & Culturas ancestrales \\
\hline 8vo lugar & Catástrofes naturales \\
\hline 9no lugar & Inseguridad \\
\hline 10mo lugar & Oportunidades de negocios \\
\hline 11er lugar & Inequidad social \\
\hline 12do lugar & Costos elevados \\
\hline 13er lugar & Insalubridad/ enfermedades \\
\hline 14to lugar & Baja conectividad aérea \\
\hline 15to lugar & Baja calidad de servicios \\
\hline
\end{tabular}

En la tabla 4 se muestran las expectativas que los turistas tenían al seleccionar Ecuador como un destino preferencial y cuáles eran los atributos que esperaban encontrar. Todas las opciones descritas fueron catalogadas bajo un rango ordinal de 1 a 7 , encontrándose que un entorno natural conservado, fue la expectativa que recibió una mayor puntuación, seguida muy de cerca por los otros atributos planteados.

Tabla 4.

Expectativas de Ecuador

\begin{tabular}{lc}
\hline Atributos & Promedio \\
\hline Entorno natural conservado & 6.12 \\
$\begin{array}{l}\text { Experiencia positiva y } \\
\text { gratificante }\end{array}$ & 6.07 \\
Patrimonio cultural único & 5.79 \\
$\begin{array}{l}\text { Servicios de alojamiento y } \\
\text { A\&B de calidad }\end{array}$ & 5.68 \\
Numerosas actividades de & 5.63 \\
ocio & \\
Buena calidad de vida & 5.59 \\
Infraestructura de calidad & 5.47 \\
\hline
\end{tabular}

La tabla 5 muestra la opinión sobre lo que el turista esperaba encontrar en el destino, para lo cual se establecieron cuatro categorías de respuestas, siendo estas: Peor de los esperaba, Lo que esperaba, Mejor de los Esperaba, No sabe/No Conoce. Donde 1 punto equivale a una respuesta negativa y 7 equivale a la máxima puntuación positiva. Como se observa en los resultados, todos los encuestados mostraron satisfacción sobre lo encontrado, puesto que el promedio de respuestas indica que el destino fue lo que esperaba o mejor de lo esperaba, a criterio de los turistas. 
Tabla 5.

Opinión sobre los que esperaba encontrar durante su visita a Ecuador.

\begin{tabular}{lcc}
\hline Atributos & & Promedio \\
\hline Conservación del entorno natural & Mejor de lo que Esperaba & 5.26 \\
Interés por el patrimonio cultural & Lo que esperaba & 4.9 \\
Calidad de vida & Mejor de lo que Esperaba & 5.07 \\
Actividades de ocio y recreación & Lo que esperaba & 5.07 \\
Calidad de infraestructura turística & Lo que esperaba & 4.71 \\
Calidad de servicio de alojamiento y A\&B & Mejor de lo que Esperaba & 5.02 \\
Experiencia en general & Mejor de lo que Esperaba & 5.45 \\
\hline
\end{tabular}

La tabla 6 muestra la frecuencia con la que los turistas han experimentado algunas emociones durante su visita al destino, en donde 1 equivale a una respuesta negativa y 7 representa la máxima puntuación positiva. Es así que la emoción "impresionado" (6.08 puntos sobre 7) es la máxima sensación percibida y "enfadado" (1.4 puntos sobre 7) es la mínima emoción percibida durante la estancia en Ecuador.

Tabla 6.

Frecuencia de la experimentación de emociones

\begin{tabular}{lc}
\hline Atributos & Promedio \\
\hline Impresionado & 6.08 \\
Encantado & 6.03 \\
Positivamente sorprendido & 6.03 \\
Contento & 5.98 \\
Indiferente & 1.99 \\
Aburrido & 1.67 \\
Desilusionado & 1.6 \\
Descontento & 1.55 \\
Enfadado & 1.4 \\
\hline
\end{tabular}

La tabla 7, muestra la intención de visitar o recomendar a Ecuador como destino, para esto se usó la misma escala numérica de preguntas anteriores, donde 1 equivale a una respuesta negativa y 7 corresponde la máxima puntuación positiva. Se observa que recomendar a Ecuador a amigos familiares es el ítem que recibió mayor puntuación. Además, es interesante destacar que la intención de regresar a Ecuador en los próximos años, es la opción que menos puntos obtuvo, pero su puntuación sigue siendo alta.

Tabla 7.

Intención de Visitar o Regresar a Ecuador

\begin{tabular}{lc}
\hline Atributos & Promedio \\
\hline Recomendar Ecuador & 6.18 \\
$\begin{array}{l}\text { Motivar a familiares/amigos para } \\
\text { que visiten Ecuador }\end{array}$ & 6.09 \\
$\begin{array}{l}\text { Nueva visita en algún momento } \\
\text { futuro }\end{array}$ & 5.33 \\
Intento regresar en próximos años & 5.19 \\
\hline
\end{tabular}


La tabla 8, muestra la valoración global de la experiencia en la visita actual al destino, con el mismo sistema de puntuación de las preguntas anteriores, donde 1 punto equivale a una respuesta negativa y 7 equivale a la máxima puntuación positiva. Es así que todas las interrogantes recibieron un promedio positivo de puntuación, siendo la "Elección acertada del destino" la que recibió una mejor calificación.

Tabla 8.

\section{Valoración global}

\begin{tabular}{lc}
\hline Atributos & Promedio \\
\hline Elección de destino acertada & 6.16 \\
$\begin{array}{l}\text { Disfrute de atractivos } \\
\text { turísticos }\end{array}$ & 6.15 \\
$\begin{array}{l}\text { Satisfacción con el servicio } \\
\text { recibido }\end{array}$ & 5.92 \\
$\begin{array}{l}\text { Es el destino turístico que } \\
\text { necesitaba }\end{array}$ & 5.75 \\
\hline
\end{tabular}

\section{Discusión}

Los datos analizados sobre el país de procedencia, evidencian una gran diversidad en cuanto a los países de origen de los turistas que usan la terminal aérea de Guayaquil. Llama la atención la notoria presencia de turistas europeos, entre ellos holandeses (45\%) y franceses $(11 \%)$, mismos que durante los días de recolección de información fueron mayoría. $\mathrm{La}$ razón de este estudio no fue la de determinar la procedencia de los turistas, sino más bien la percepción sobre el destino Ecuador, por tal motivo no se puede determinar a ciencia cierta porque existió un porcentaje tan grande de turistas provenientes de los Países Bajos durante la etapa de recolección de datos. Por otra parte, es importante mencionar que desde hace varias décadas existe una frecuencia aérea directa entre Guayaquil y Ámsterdam, convirtiéndose, el aeropuerto de Guayaquil en último punto de salida del Ecuador hacia la parte norte de Europa, por lo que la consolidación de esta frecuencia podría justificar el alto número de turistas provenientes de esta nacionalidad.

Así mismo llama la atención que según datos del Ministerio de Turismo del Ecuador (2016), los turistas norteamericanos representan el principal grupo de visitantes hacia el país, sin embargo, este hecho no coincidió con lo obtenido durante la investigación de campo, puesto que el grupo de estadounidenses (6\%) era minoritario con respecto a otras nacionalidades. Actualmente, Guayaquil posee tres conexiones directas hacia Estados Unidos: una hacia Miami, otra hacia Fort Lauderdale y la última hacia Nueva York; sin embargo, el otro aeropuerto internacional del Ecuador, es decir, el de la ciudad de Quito, posee las mismas rutas, pero adicionalmente oferta vuelos directos hacia Houston y Atlanta, por lo que sería interesante realizar futuros estudio, sobre la ruta de ingreso al país que usan los norteamericanos como principal mercado del Ecuador.

Al observar los datos sobre visitas anteriores al Ecuador, sólo el 14\% de encuestados tenían una experiencia previa en el país como visitante y el 86\% venía a Ecuador por primera vez. Kozak (2001) utiliza la palabra en inglés "repeaters" para referirse a los visitantes repetidores de un destino, por lo que en adelante a efectos de este estudio se usará el 
término propuesto por el mencionado autor, quien sostiene que los turistas repetidores son más propensos a volver varias veces a los mismos destinos en el futuro, por cuanto tuvieron experiencias significativas en el lugar visitado. Por tanto, se convierte en un factor determinante para que un turista se vuelva repetidor, que experimente sensaciones positivas $\mathrm{y}$ tengan una buena autopercepción de su viaje realizado. La siguiente figura repre- senta las emociones que experimentaron los turistas repetidores de Ecuador como destino turístico. Esta variable como ya se mencionó fue medida con una escala ordinal del 1 al 7 , donde este último número era la mayor puntuación posible. Es así que las emociones positivas: contento, encantado, positivo e impresionado recibieron una mayor valoración que las negativas.

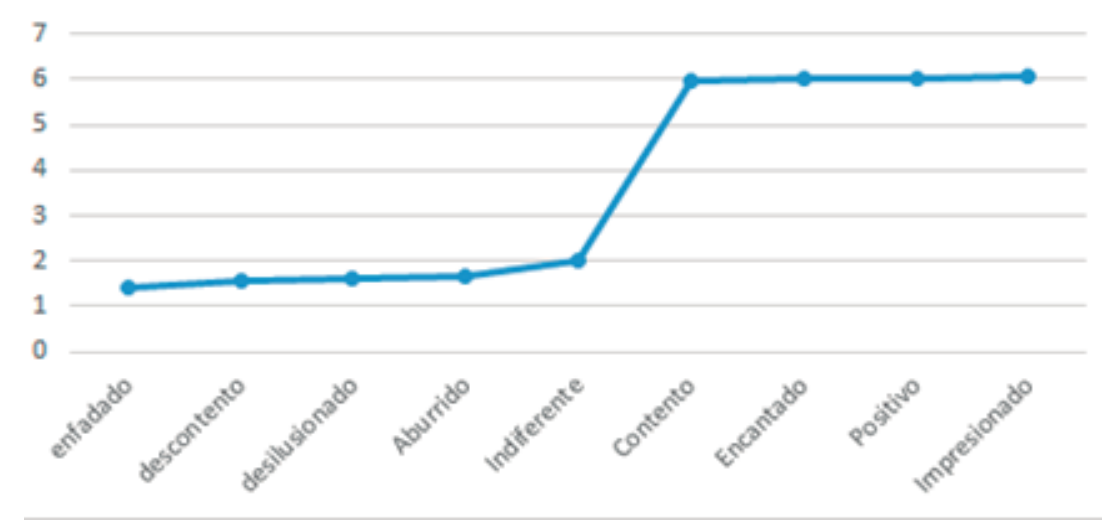

Figura 2. Correlación de la experimentación de emociones en los turistas repetidores

En cuanto al gasto turístico, el nivel promedio por persona oscila entre $1000 \mathrm{y}$ 3000 dólares (46\%), así mismo un grupo importante gastó menos de 1000 dólares por persona $(40 \%)$ y un pequeño grupo indicó que gastaba más de 5000 dólares (3\%). En cuanto a la estancia, la mayor parte de los encuestados indicó permanecer aproximadamente 2 semanas (51\%), seguido por los que indicaron permanecer en promedio 3 semanas (23\%). En cualquiera de los casos si se compara la cantidad gastada con la duración de la estancia, el gasto diario por persona parecería ser limitado. los turistas que más gasto hicieron en el país, se observa que los visitantes de China, Francia, algunos países de Latinoamérica y Estados Unidos son los que presentan una mayor cantidad de gasto durante su visita, aunque curiosamente el grupo de turistas holandeses y alemanes es más numeroso que los de otras nacionalidades.

Tomando en cuenta los datos sobre 


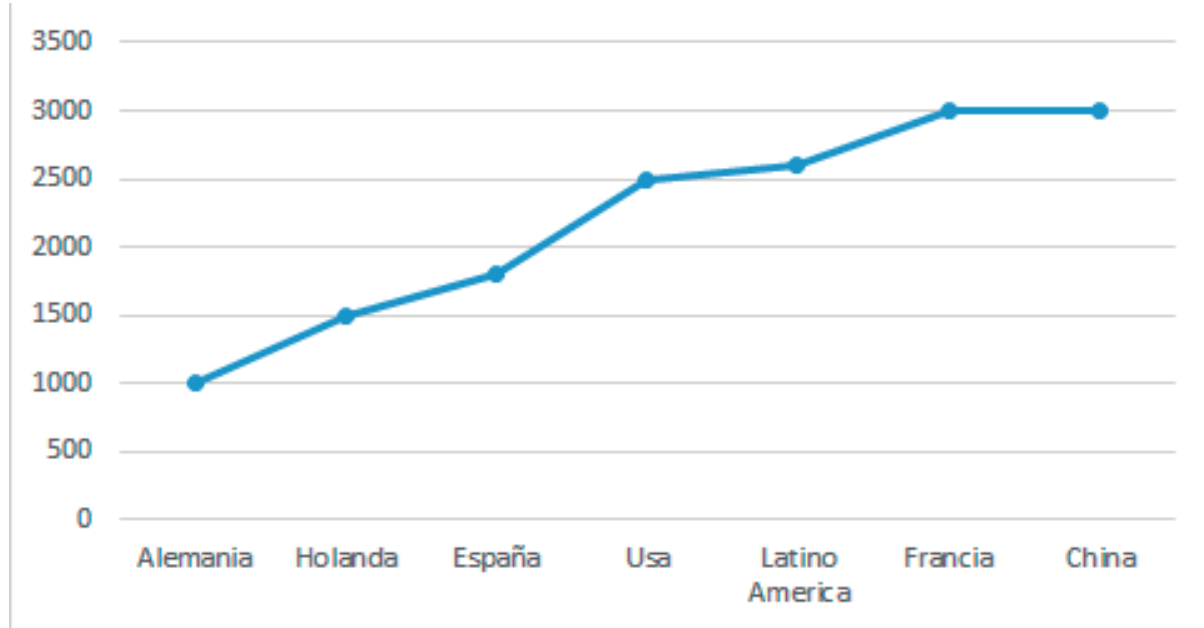

Figura 3. Correlación de la nacionalidad de los turistas con su nivel de gasto en el destino, donde el eje " $y$ " representa la cantidad de dinero en dólares estadounidenses (USD).

Al analizar las preferencias de los turistas que más gastan, esto es chinos, franceses y latinoamericanos, se puede observar que los turistas chinos prefieren los entornos naturales conservados, los franceses el patrimonio cultural mientras que los latinoamericanos, las actividades de ocio y recreación.

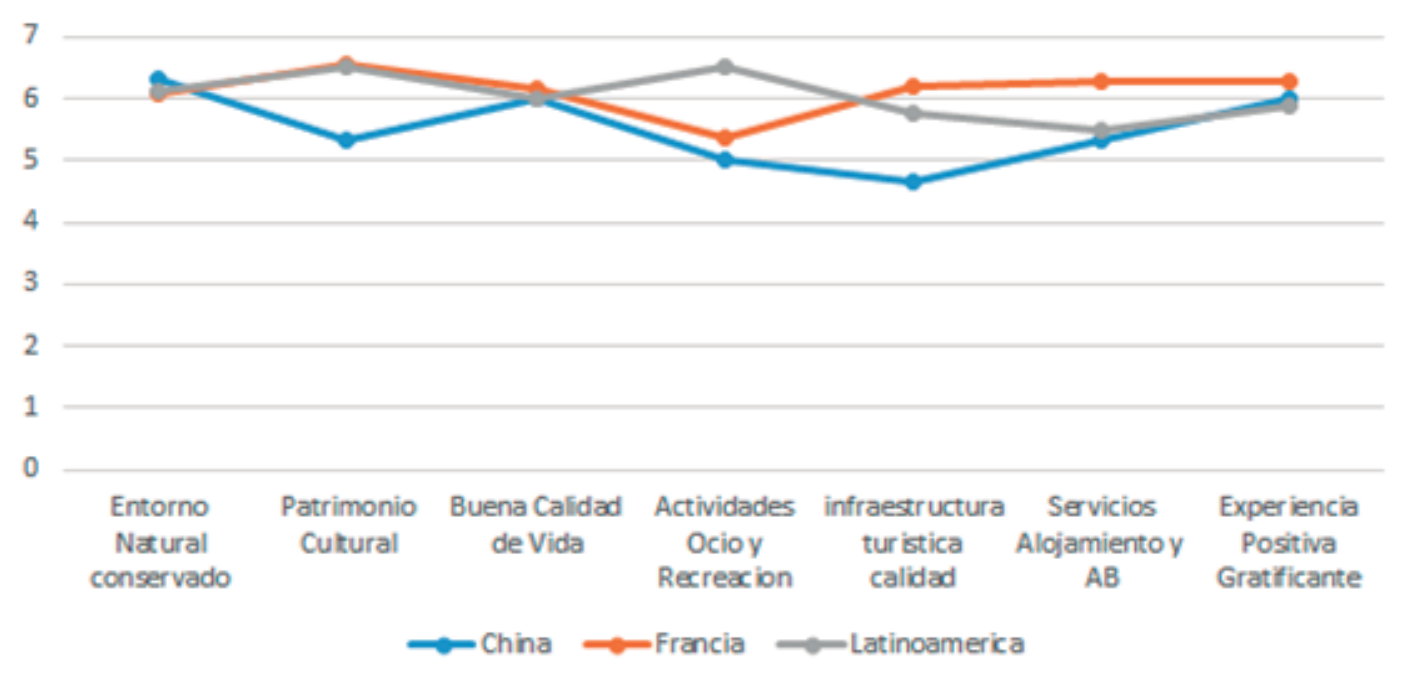

Figura 4. Correlación de las preferencias mostradas en los turistas con mayor nivel de gasto en el destino. 
Por otro lado, es interesante la asociación que el turista hace del país previo a su visita con las características que piensa encontrar, destacándose con una mayor puntuación los paisajes únicos, la flora y fauna y los entornos de sol y playa. Así mismo llama la atención que el turista tenía la expectativa de llegar a Ecuador y encontrar un entorno natural conservado $y$ tener una experiencia positiva y gratificante. Luego de la visita efectuada, esta investigación comprobó que la experiencia del visitante fue considerablemente buena, tal como lo muestran los datos recogidos sobre la interrogante de elección del destino. Así mismo, muchos de ellos estarían interesados en "Recomendar a Ecuador" y a "Motivar a familiares o amigos a que lo visiten", según lo indican los datos obtenidos en estas variables.

\section{Conclusiones}

Esta investigación ha utilizado diferentes variables para recopilar información relevante a la percepción de Ecuador como destino turístico, así mismo se analizó el país de procedencia de los visitantes. Según datos del Ministerio de Turismo, son norteamericanos el principal país emisor de turistas de larga distancia para el Ecuador, sin embargo, al momento de realizar la investigación de campo, los datos determinaron que había una mayor cantidad de turistas de diversos países europeos, como Holanda, Francia, España y Alemania. . Esto no significa que este estudio haya demostrado que los datos oficiales no son valederos, sino más bien que durante la toma de datos, los turistas de estas naciones eran eran mayoría. Considerando la estacionalidad del turismo, se recomienda que futuras investigaciones realicen la recopilación de información en diferentes épocas del año para obtener diferentes espectros.

En temas de gastos se obtuvo que los turistas encuestados en su mayoría gastan entre 1000 y 3000 dólares por 2 semanas de estadía en promedio, esto indicaría que la cantidad de gastos para el número de días es aún baja, más si se considera que el Ecuador ofrece servicios variados como venta de artesanías, gastronomía, souvenirs y alojamiento de diferente categoría que incentivan el consumo de los visitantes.

Entre el origen de los turistas con mayor nivel de gasto en el país se encuentra que los latinoamericanos se posicionan en tercer lugar, superando a varios países de Europa e inclusive a los estadounidenses; Francia y China ocupan el segundo y primer lugar, respectivamente. Los tres grupos que encabezan el nivel de gasto turístico en el Ecuador coinciden en que el entorno natural, la buena calidad de vida y la experiencia gratificante son importantes para ellos. Así mismo, los tres grupos coinciden en que la "Experiencia positiva y gratificante" es de alta. Sin embargo, los holandeses y alemanes que son el mayor grupo de visitantes prefieren un "Ambiente natural conservado".

La mayoría de los turistas coinciden que Ecuador fue una elección acertada como destino, pero pocos consideran regresar al país en un momento futuro. 
A pesar de esto, casi la totalidad de los entrevistados coinciden en recomendar a sus familiares y amigos, el país. Estas opiniones contradictorias están muy ligadas a la esencia del turismo y al factor moda, pues los turistas suelen cambiar de destino de vacaciones cada año, sin que esto signifique que la elección del destino no haya sido apropiada.

Por otra parte, los datos indican que los encuestados prefieren visitar entornos naturales, flora y fauna y sitios patrimoniales, donde quizá la oferta de servicios es limitada o los costos son aún muy bajos, lo que daría como consecuencia también consumos de poco gasto. Más si se considera, que la entrada a parques nacionales y museos públicos es gratuita, salvo el caso del Parque Nacional Galápagos y el Yasuní, que cobran un valor considerable para su ingreso.

Sobre los turistas frecuentes o repetidores y su relación con la experimentación de emociones, los hallazgos muestran que la mayoría de éstos concibieron pensamientos gratificantes hacia Ecuador como destino turístico, siendo esto un factor determinante para repetir su visita al país. Sin embargo, tan solo una quinta parte de los encuestados ha repetido el destino, por lo que tampoco debería esperarse que un gran porcentaje del restante grupo de encuestados y que también ha experimentado emociones positivas en su visita, regrese al país en un futuro.

\section{Referencias}

Barrantes, R. (2000). Investigación: un camino al conocimiento. Un enfo- que cuantitativo y cualitativo. San José: Universidad Estatal a Distancia.

Bryman, A. (2012). Social Research Methods 4th edition. Oxford: Oxford University Press.

Castells, M., (2011). The Rise of the Network Society: The Information Age: Economy, Society, and Culture.

Castillo, E. (2015). El turismo en Ecua$d o r$, Nuevas tendencias en el turismo sostenible y contribución al crecimiento económico. Revista Galega de Economía, 24, 2.

Chen, C. F., \& Phou, S. (2013). A closer look at destination: Image, personality, relationship and loyalty. Tourism Management 36, 269-278.

Cruz, A. (2017). Plan de marketing city como instrumento de apoyo a la gestión del fortalecimiento de la identidad e imagen de Guayaquil. Recuperado de http://repositorio.utmachala.edu.ec/handle/48000/10150

Delgado, A. (2013). Guayaquil. Cities, $31,515-532$.

Delgado, M. \& Chávez, A. (2016). Status of lifelong learning in Guayaquil, Ecuador. Innova Research Journal, 1(10), 121 - 133.

Díaz, O. (2015). Recent developmen 
and current relevance of airport commercial revenues Cali: Universidad ICESI

Dirección General de Aviación Civil del Ecuador (2017). Visión, misión y valores. Mayo 15, 2017, Recuperado de DGAC http://www.aviacioncivil.gob.ec/?p=1356

Di Marino, E. (2008). The Strategic Dimension of Destination Image. an Analysis of the French Riviera Image from the Italian Tourist' Perceptions. $17 \mathrm{~h}$ International Tourism and Leisure Symposium.

El Telégrafo. (2015). Ecuador tiene cinco cinco mercados objetivos para promocionar y captar el turismo. El Telégrafo. Recuperado de http://www.eltelegrafo.com.ec/noticias/economia/8/ecuador-tiene-cinco-mercados-objetivos-para -promocionar-y-captar-el-turismo

Ferrando, M. G. (2000). El análisis de la realidad social. Métodos y técnicas de investigación. Madrid: Alianza Universidad.

Jiménez, E., Claro, J., \& Pinho de Sousa, J. (2014). The airport business in a competitive environment. Procedia - Social and Behavioral Sciences 111, 947-954

Kozak, M. (2001). Repeaters behavior at two distinct destinations. Annals of Tourism Research, 28 (3), 784-807.
Law, R., Leung, R., Lo, A., Leung, D., \& Hoc Nan Fong, L. (2015). Distribution channel in hospitality and tourism: Revisiting disintermediation from the perspectives of hotels and travel agencies, International Journal of Contemporary Hospitality Management, 27 (3), pp.431-452

Leclerc, L. (2013, agosto 8). Aeropuertos, eje de desarrollo económico. La República, 1 de enero de 2017. Obtenido de http://www.listindiar i o . c o m / 1 a - r e p u b 1 i $\mathrm{ca} / 2013 / 08 / 08 / 287535 / \mathrm{ae}$ ropuertos-ejes-de-desarrollo-econ $\% \mathrm{C} 3 \% \mathrm{~B} 3$ mico Base de datos.

Ministerio de Turismo (2016). Boletín Mensual 2015-2016. Obtenido de http://servicios.turismo.gob.ec/index.php/portfolio/turis mo-cifras/19-inteligencia-de-merc ados/boletin-mensual/95

Ministerio de Turismo. (2008). Ley de Turismo. Obtenido de h t t p : / / w w w.t u ris m o.gob.ec/wp-content/uploads/downloads/2014/02/Ley-de-Turismo-M INTUR.pdf

Miralles, J. (2015). Efecto de la Relación de Compresión en el Rendimiento de Motores de Combustión Interna a diferentes Altitudes. Valencia: Universidad CEU Cardenal Herrera

Mohd, N., Abdul, N., \& Peck Leong, T. (2016, Abril 9-10). A stakeholder 
Analysis of the klia2 Airport Terminal Project. Environment Behavior, 7th Asian conference on Environment Studies, 9.

Morosan, C. and Jeong, M. (2008), "Users'perceptions of two types of hotel reservation Websites", International Journal of Hospitality Management, Vol. 27 No. 2, pp. 284-292.

Ministerio de Relaciones exteriores y movilidad humana. (2015). Ley orgánica de servicio exterior. enero, 2017, de Sitio web: http://www.cancilleria.g o b . e c / w p - c on te n t / u ploads/2015/04/LEY-ORGANICA -DE-SERVICIO-EXTERIOR.pdf

Organización Mundial del Turismo.

(10 de diciembre de 2016). Sobre el turismo. Obtenido de http://www2.unwto.org/es

Peralta, J. (2013). Evaluación energética y Análisis Estadístico del régimen de Vientos del perfil costero ecuatoriano. La Plata: Congreso Iberoamericano de Ingeniería Mecánica.

Rajesh, R. (2013). Impact of Tourist perceptions, destination image and tourist satisfaction on destination loyalty: A conceptual model. Pasos, 11 (3), pp. 67-78.

San Martín Gutiérrez, H. (2006). Estudio de la imagen de destino turístico y el proceso global de satisfacción: adopción de un enfoque integrador. Universidad de Cantabria.

Thakran, K., \& Verma, R. (2013) The Emergence of Hybrid Online Distribution Channels in Travel, Tourism and Hospitality. Cornell Hospitality Quaaterly, 54(3), 240-247. Recuperado de http://scholarship.sha.cornell.edu/articles/46/

The Boeing Company. (2014). 787 Airplane Characteristics for Airport Planning

United Nations Development Programme. (2013). Obtenido el 16 de mayo del 2017 de UNDP http://www.undp.org/content/undp/en/home.html

Velasco González, M. (2009). Gestión turística del patrimonio cultural: enfoques para un desarrollo sostenible del turismo cultural. Cuadernos de Turismo, 23, pp. 237- 253

Xiang, Z., Magnini, V., \& Fesenmaier, D.R. (2014). Information technology and consumer behavior in travel and tourism: Insights from travel planning using the internet. Journal of Retailing and Consumer Services 22, pp. 244-249

Zhang, H., Xiaoxiao, F., Liping, A., \& Lin, L. (febrero, 2014). Destination image and tourist loyalty: A meta-analysis. Tourism Management, 40, pp. 213-223. 